\title{
A Robust State Feedback Adaptive Controller with Improved Transient Tracking Error Bounds for Plants with Unknown Varying Control Gain
}

\author{
A. Rincon 1 , F. Angulo 2 and G. Osorio ${ }^{2}$ \\ 1 Universidad Católica de Manizales \\ 2Universidad Nacional de Colombia - Sede Manizales - Facultad de Ingeniería y \\ Arquitectura - Departamento de Ingeniería Eléctrica, Electrónica y Computación - \\ Percepción y Control Inteligente - Bloque Q, Campus La Nubia, Manizales,
}

Colombia

\section{Introduction}

The design of robust model reference adaptive control (MRAC) schemes for plants in controllable form, comprising unknown varying but bounded coefficients and varying control gain has attracted a great deal of research. Many nonlinear systems may be described by the controllable form; for instance, second order plants (see (Hong \& Yao, 2007), (Hsu et al., 2006), (Yao \& Tomizuka, 1994), (Jiang \& Hill, 1999)) and systems whose nonlinear behavior or part of it, is represented by some function approximation technique (cf. Nakanishi et al. (2005), (Chen et al., 2008), (Tong et al., 2000), (Huang \& Kuo, 2001), (Yousef \& Wahba, 2009), (Hsu et al., 2006), (Koo, 2001), (Labiod \& Guerra, 2007)).

The state adaptive backstepping (SAB) of (Kanellakopoulos et al., 1991) is a common framework for the design of adaptive controllers for plants in controllable form. As is well known, a major difficulty in introducing robustness techniques to SAB based schemes is that the states $z_{i}$ and the stabilizing functions must be differentiable to certain extent (see (Yao \& Tomizuka, 1997), (Yao, 1997), (Ge \& Wang, 2003)).

The robust SAB scheme of (Zhou et al., 2004), (Su et al., 2009), (Feng, Hong, Chen \& Su, 2008) has the advantage that the knowledge on the upper or lower bounds of the plant coefficients can be relaxed if the controller is properly designed and the control gain is constant or known. The approach is based on the truncation method of (Slotine \& Li, 1991), pp. 309. The stabilizing functions are smoothed at each $i$-th step in order to render it differentiable enough. The following benefits are obtained: i) the scheme is robust with respect to unknown varying but bounded coefficients, ii) upper or lower bounds of the plant coefficients are not required to be known, and iii) the tracking error converges to a residual set whose size is user-defined.

The specific case of unknown varying control gain is an important issue, more difficult to handle than other unknown varying coefficients. The varying control gain is usually handled by means of robustness techniques (cf. (Wang et al., 2004), (Huang \& Kuo, 2001), (Bechlioulis 
\& Rovithakis, 2009), (Li et al., 2004)) or the Nussbaum gain technique (cf. (Su et al., 2009), (Feng, Hong, Chen \& Su, 2008), (Feng et al., 2006), (Ge \& Wang, 2003)). The above methods are applicable to plants in parametric-pure feedback or controllable form, and with controllers that use the SAB or the MRAC as the control framework.

In (Wang et al., 2004), a system with dead zone in the actuator is considered, assuming that both dead zone slopes have the same value. The input term is rewritten as the sum of an input term with constant control gain plus a bounded disturbance-like term. The disturbance term is rejected by means of a robust technique, based on (Slotine \& Li, 1991) pp. 309. Nevertheless, this strategy is not valid for different values of the slopes. Other robustness techniques comprise a control law with compensating terms and either a projection modification of the update law, as in cf. (Huang \& Kuo, 2001), or a $\sigma$ modification as in (Bechlioulis \& Rovithakis, 2009), (Li et al., 2004). Nevertheless, some lower or upper bounds of the plant coefficients are required to be known.

The Nussbaum gain technique can relax this requirement, as can be noticed from (Su et al., 2009), (Feng, Hong, Chen \& Su, 2008), (Feng, Su \& Hong, 2008). The main drawbacks of the Nussbaum gain method are (see (Su et al., 2009), (Feng, Hong, Chen \& Su, 2008), (Feng et al., 2006), (Ge \& Wang, 2003), (Feng et al., 2007), (Feng, Su \& Hong, 2008), (Ren et al., 2008), (Zhang \& Ge, 2009), (Du et al., 2010)): i) the upper bound of the transient behavior of the tracking error is significantly modified in comparison with that of the disturbance-free case: the value of this bound depends on the time integral of terms that comprise Nussbaum terms, and ii) the controller involves an additional state, which is necessary to compute the Nussbaum function.

Other drawbacks are: i) the control gain is assumed to be the product of a unknown constant and a known function, as in (Tong et al., 2010), (Liu \& Tong, 2010), ii) the control gain is assumed upper bounded by some unknown constant, as in (Zhang \& Ge, 2009), (Du et al., 2010), (Su et al., 2009), (Feng, Hong, Chen \& Su, 2008), (Feng et al., 2006), (Ge \& Wang, 2003), (Feng et al., 2007), (Feng, Su \& Hong, 2008), (Ren et al., 2008), iii) the control gain is assumed upper bounded by a known function, as in (Ge \& Tee, 2007), (Psillakis, 2010), iv) upper or lower bounds of the plant coefficients are required to be known to achieve asymptotic convergence of the tracking error to a residual of user-defined size, as in (Ge \& Tee, 2007), (Chen et al., 2009), (Feng et al., 2006), (Ge \& Wang, 2003), (Feng et al., 2007), (Ren et al., 2008), (Ge \& Tee, 2007), (Tong et al., 2010), (Liu \& Tong, 2010), iv) the control or update law involves signum type signals, as in (Zhang \& Ge, 2009), (Du et al., 2010), (Psillakis, 2010), (Su et al., 2009), (Feng, Hong, Chen \& Su, 2008), (Feng, Su \& Hong, 2008).

Recent adaptive control schemes based on the direct Lyapunov method achieve improved transient performance. For instance, $L_{1}$ adaptive control, with the drawback that the control gain is assumed constant, as in (Cao \& Hovakimyan, 2006), (Cao \& Hovakimyan, 2008a), (Cao \& Hovakimyan, 2008b), (Dobrokhodov et al., 2008), (Li \& Hovakimyan, 2008).

Other works have the following drawbacks:

i) The control gain is assumed constant, as in (Zhou et al., 2009), (Wen et al., 2009), (Bashash \& Jalili, 2009).

ii) The control gain is assumed upper bounded by some unknown constant, as in (Chen, 2009), (Ho et al., 2009) and (Park et al., 2009). 
iii) The control gain is assumed upper bounded by some known function as in (Bechlioulis \& Rovithakis, 2009).

iv) Upper or lower bounds of plant parameters are required to be known to achieve asymptotic convergence of the tracking error to a residual set of user-defined size, as in (Bashash \& Jalili, 2009), (Chen, 2009), (Ho et al., 2009), (Park et al., 2009) and (Bechlioulis \& Rovithakis, 2009).

In this chapter, we develop a controller that overcomes the above drawbacks, so that:

Bi) The upper bound of tracking error transient value does not depend on time integral terms.

Bii) Additional states are not used in the controller.

Biii) The control gain is not required to be upper bounded by a constant.

Biv) The control gain is not required to be bounded by a known function.

Bv) Upper or lower bounds of the plant parameters are not required to be known.

Bvi) The control and update laws do not involve signum type signals.

Bvii) The tracking error converges to a residual set whose size is user-defined.

We consider systems described by the controllable form model with arbitrary relative degree, unknown varying but bounded coefficients and varying control gain. We use the $\mathrm{SAB}$ of (Kanellakopoulos et al., 1991) as a basic framework for the control design, preserving a simple definition of the states resulting from the backstepping procedure. We use the Lyapunov-like function method to handle the unknown time varying behavior of the plant parameters. All closed loop signals remain bounded so that parameter drifting is prevented.

The key elements to handle the varying behavior of the control gain are: i) introduce the control gain in the term involving the adjusted parameter vector, by means of the inequality that relates the control gain and its lower bound, and ii) apply the Young's inequality.

In current works that deal with plants in controllable form and time varying parameters and use the state transformation based on the backstepping procedure, they modify the defined states at each step of the state transformation in order to tackle the unknown time varying behavior of the plant parameters. Instead of altering the state transformation, we formulate a Lyapunov-like function, such that its magnitude and time derivative vanish when the states resulting from the state transformation reach a target region.

The control design and proof of boundedness and convergence properties are simpler in comparison to current works that use the Nussbaum gain method. The controller is also simpler as it does not introduce additional states that would be necessary to handle the unknown time varying control gain.

The chapter is organized as follows. In section 2 we detail the plant model. In section 3 we present the goal of the control design. In section 4 we carry out a state transformation, based on the state backstepping procedure. In section 5 we derive the control and update laws. In section 6 we prove the boundedness of the closed loop signals. In section 7 we prove the convergence of the tracking error $e$, finally, in section 8 we present an example. 


\section{Problem statement}

In this section we detail the plant and the reference model. Consider the following plant in controllable form:

$$
y^{(n)}=\gamma_{n}^{\top} a+b u+d
$$

where $y(t) \in \mathbb{R}$ is the system output, $u(t) \in \mathbb{R}$ the input, $a$ a vector of varying entries, $\gamma_{n}$ a known vector, $b$ the control gain, and $d$ a disturbance-like term. We make the following assumptions:

Ai) The vector $a$ involves unknown, time varying, bounded entries $a_{1}, \cdots, a_{j}$, which satisfy: $\left|a_{1}\right| \leq \bar{\mu}_{1}, \cdots,\left|a_{j}\right| \leq \bar{\mu}_{j}$, where $\bar{\mu}_{1}, \cdots, \bar{\mu}_{j}$ are unknown, positive constants.

Aii) The entries of the vector $\gamma_{n}$ are known linear or nonlinear functions of $y, \cdots, y^{(n-1)}$.

Aiii) The terms $y, \dot{y}, \cdots, y^{(n-1)}$ are available for measurement.

Aiv) The term $d$ represents either external disturbances or unknown model terms that satisfy:

$$
|d| \leq \mu_{d} f_{d}
$$

where $\mu_{d}$ is an unknown positive constant, and $f_{d}$ is a known function that depends on $y, \cdots$, $y^{(n-1)}$. In the case that $d$ is bounded, we have $f_{d}=1$. The term $d$ may come from the product of a known function $g_{d}$ with an unknown varying but bounded coefficient $c_{g}$ : $d=c_{g} g_{d}$, $\left|c_{g}\right| \leq \mu_{d}$, so that $f_{d}=\left|g_{d}\right|$, where $\mu_{d}$ is an unknown positive constant whereas $g_{d}$ is a known function.

Av) The control gain $b$ satisfies:

$$
|b| \geq b_{m}>0, b \neq 0 \forall t \geq t_{0}
$$

where $b_{m}$ is an unknown lower bound, and the value of the signum of $b$ is constant and known.

Remark. We recall that $\mu_{d}, b_{m}, \bar{\mu}_{1}, \cdots, \bar{\mu}_{j}$ are unknown constants. In contrast, the values of $y$, $\cdots, y^{(n-1)}, \gamma_{n}, f_{d}, \operatorname{sgn}(b)$ are required to be known. Notice in assumption Av that we do not require the control gain $b$ to be upper bounded by any constant. That is a major contribution with respect to current works that use the Nussbaum gain method, e.g (Su et al., 2009), (Feng, Hong, Chen \& Su, 2008), (Feng, Su \& Hong, 2008), (Feng et al., 2007), (Ge \& Wang, 2003). The requirement about the value of the signum of $b$ is a common and acceptable requirement.

\section{Control goal}

Let

$$
\begin{aligned}
& e(t)=y(t)-y_{d}(t) \\
& y_{d}^{(n)}+a_{m, n-1} y_{d}^{(n-1)}+\cdots+a_{m, o} y_{d}=a_{m, o} r \\
& \Omega_{e}=\left\{e:|e| \leq C_{b e}\right\}
\end{aligned}
$$


where $e(t)$ is the tracking error, $y_{d}(t)$ is the desired output, $\Omega_{e}$ is a residual set, $r$ is the reference signal. Moreover, $a_{m, n-1}, \cdots, a_{m, o}$ are constant coefficients defined by the user, such that the polynomial $K(p)$ is Hurwitz, being $K(p)$ defined as $K(p)=p^{(n)}+a_{m, n-1} p^{(n-1)}+\cdots+$ $a_{m, o}$. The reference signal $r(t)$ is bounded and user-defined. The constant $C_{b e}$ is positive and user-defined.

The objective of the MRAC design is to formulate a controller, provided by the plant model (1) subject to assumptions Ai to Av, such that:

i) The tracking error $e$ converges asymptotically to the residual set $\Omega_{e}$.

ii) The control signals are bounded and do not involve discontinuous signals.

\section{State transformation based on the state backstepping}

In this section we carry out a state transformation by following the steps $0, \cdots, n$ of the backstepping procedure. The plant model (1) can be rewritten as follows:

$$
\begin{aligned}
& \dot{x}_{i}=x_{i+1}, \quad 1 \leq i \leq n-1 \\
& \dot{x}_{n}=a^{\top} \gamma_{n}\left(x_{1}, \cdots, x_{n}\right)+b u+d \\
& x_{1}=y, x_{2}=\dot{y}, \cdots, x_{n}=y^{(n-1)}
\end{aligned}
$$

The model $(7,8)$ can be obtained by making $\gamma_{1}=\cdots=\gamma_{n-1}=0$ in the parametric - pure feedback form of (Kanellakopoulos et al., 1991). We use the SAB of (Kanellakopoulos et al., 1991 ) as the basic framework for the formulation of the control and update laws.

We develop the SAB for the plant model $(7,8)$, and introduce a new robustness technique. Since the order of the plant is $n$, the procedure comprises the steps $0, \cdots, n$, to be carried out in a sequential manner.

Step 0 . We begin by defining the state $z_{1}$ as the tracking error:

$$
z_{1}=e=y-y_{d}=x_{1}-y_{d}
$$

Step $i(1 \leq i \leq n-1)$. At each $i$-th step, we obtain the dynamics of the state $z_{i}$ by deriving it with respect to time, and using the definitions of $\dot{x}_{i+1}$ provided by (7). For the sake of clarity, we develop the step 1 and then we state a generalization for $(1 \leq i \leq n-1)$.

For the case $i=1$, we differentiate $z_{1}$ defined in (9) and use the definition of $\dot{x}_{1}$ provided by (7) with $i=1$ :

$$
\begin{aligned}
& \dot{z}_{1}=\dot{x}_{1}-\dot{y}_{d}=x_{2}-\dot{y}_{d}=x_{2}+\varphi_{1} \\
& \varphi_{1}=\varphi_{1}\left(\dot{y}_{d}\right)=-\dot{y}_{d}
\end{aligned}
$$

where $\varphi_{1}$ is a known function of $\dot{y}_{d}$. Equation (10) can be rewritten as:

$$
\begin{aligned}
& \dot{z}_{1}=-c_{1} z_{1}+z_{2} \\
& z_{2}=x_{2}+c_{1} z_{1}-\dot{y}_{d}
\end{aligned}
$$

where $c_{1} \geq 2$ is a positive constant of the user choice. The dynamic equation of $z_{2}$ is obtained by differentiating it with respect to time. The same procedure must be followed until the step 
$i=n-1$. To state a generalization, we express $\dot{z}_{i}$ as:

$$
\begin{aligned}
\dot{z}_{i} & =x_{i+1}+\varphi_{i}, \\
\varphi_{i} & =\varphi_{i}\left(z_{1}, \cdots z_{i}, y_{d}, \dot{y}_{d}, \cdots, y_{d}^{(i)}\right),
\end{aligned}
$$

where $\varphi_{i}$ is a known scalar term, that is function of $z_{1}, \cdots, z_{i}, y_{d}, \dot{y}_{d}, \cdots, y_{d}^{(i)}$. Equation (13) can be rewritten as:

$$
\begin{aligned}
& \dot{z}_{i}=-c_{i} z_{i}+z_{i+1} \\
& z_{i+1}=x_{i+1}+\varphi_{i}+c_{i} z_{i}
\end{aligned}
$$

where $c_{i} \geq 2$ is a positive constant of the user choice. At the step $i=n-1$ we obtain the dynamic equation for $z_{n-1}$ and the expression for $z_{n}$ as indicated by $(15,16)$.

Remark. Notice that the definition of the states $z_{i}$ is similar to that of the disturbance free case, so that a simple design is preserved. This is due to the following facts:

i) Disturbance like terms are absent in the dynamics $\dot{x}_{1}, \cdots, \dot{x}_{n-1}$ given by (7), so that they are also absent in the dynamics $\dot{z}_{1}, \cdots, \dot{z}_{n-1}$, as can be noticed in (13).

ii) Dead zone functions of the states $z_{i}$ are not used.

Step $n$. We obtain the dynamics of $z_{n}$ by differentiating it with respect to time and using the expression of $\dot{x}_{n}$ provided by (8):

$$
\begin{aligned}
& \dot{z}_{n}=b u+a^{\top} \gamma_{n}+\varphi_{n}+d \\
& \varphi_{n}=\varphi_{n}\left(z_{1}, \cdots, z_{n}, y_{d}, \cdots, y_{d}^{(n)}\right)
\end{aligned}
$$

where $\varphi_{n}$ is a known scalar that is function of $z_{1}, \cdots, z_{n}, y_{d}, \cdots, y_{d}^{(n)}$. Notice that the disturbance like term $d$ and the control input $u$ appear explicitly at the dynamics of $z_{n}$, at the step $n$ of the procedure. Thus, we have completed the state transformation, which allows us to develop the controller.

\section{Control and update laws}

In this section we develop the control and update laws, taking into account the assumptions stated in section 2 and the goals of section 3 . The key elements of the procedure are:

i) Incorporate the assumptions $\mathrm{Ai}$ and Aiv, concerning the unknown time varying parameter $a_{1}, \cdots, a_{j}$ and the disturbance like term $d$.

ii) Carry out a linear parameterization.

iii) Express the parameterization in terms of adjustment error and adjusted parameter vector.

iv) Introduce the control gain $b$ within the adjusted parameter vector.

v) Formulate the control law.

vi) Formulate a Lyapunov-like function and find its time derivative. 
vii) Formulate the update law.

We begin by rewriting (17) as follows:

$$
\dot{z}_{n}=-c_{n} z_{n}+b u+a^{\top} \gamma_{n}+\varphi_{n}+c_{n} z_{n}+d,
$$

where $c_{n} \geq 2$ is a positive constant of the user choice. Multiplying (19) by $z_{n}$, we obtain:

$$
z_{n} \dot{z}_{n}=-c_{n} z_{n}^{2}+b z_{n} u+z_{n} a^{\top} \gamma_{n}+z_{n}\left(\varphi_{n}+c_{n} z_{n}\right)+z_{n} d
$$

The term $z_{n} a^{\top} \gamma_{n}+z_{n}\left(\varphi_{n}+c_{n} z_{n}\right)+z_{n} d$ can be rewritten as follows:

$$
\begin{aligned}
& z_{n} a^{\top} \gamma_{n}+z_{n}\left(\varphi_{n}+c_{n} z_{n}\right)+z_{n} d=z_{n}\left(a^{\top} \gamma_{n}+d+\varphi_{n}+c_{n} z_{n}\right) \\
& \quad=z_{n}\left(a_{[1]} \gamma_{n[1]}+\cdots+a_{[j]} \gamma_{n[j]}+d+\varphi_{n}+c_{n} z_{n}\right) \\
& \quad \leq\left|z_{n}\right|\left(\left|a_{[1]} \gamma_{n[1]}\right|+\cdots+\left|a_{[j]} \gamma_{n[j]}\right|+|d|+\left|\varphi_{n}+c_{n} z_{n}\right|\right)
\end{aligned}
$$

using assumptions Ai and Aiv of section 2 and parameterizing, we obtain:

$$
\begin{aligned}
& z_{n} a^{\top} \gamma_{n}+z_{n}\left(\varphi_{n}+c_{n} z_{n}\right)+z_{n} d \\
& \quad \leq\left|z_{n}\right|\left(\bar{\mu}_{1}\left|\gamma_{n[1]}\right|+\cdots+\bar{\mu}_{j}\left|\gamma_{n[j]}\right|+\mu_{d} f_{d}+\left|\varphi_{n}+c_{n} z_{n}\right|\right) \\
& \quad=\sqrt{b_{m n}}\left|z_{n}\right| \bar{\varphi}^{\top} \theta
\end{aligned}
$$

where

$$
\begin{aligned}
\bar{\varphi} & =\left[\left|\gamma_{n[1]}\right|, \cdots,\left|\gamma_{n[j]}\right|, f_{d},\left|\varphi_{n}+c_{n} z_{n}\right|\right]^{\top} \\
\theta & =\left(1 / \sqrt{b_{m n}}\right)\left[\bar{\mu}_{1}, \cdots, \bar{\mu}_{j}, \mu_{d}, 1\right]^{\top}
\end{aligned}
$$

Notice that the entries of the vector $\theta$ are unknown, positive, constant, because bounds of the time varying parameters $a_{i}$ and $d$ have been introduced, according to the properties in assumptions Ai and Aiv of section 2. Now, we express (22) in terms of adjustment error and adjusted parameter vector:

$$
z_{n} a^{\top} \gamma_{n}+z_{n}\left(\varphi_{n}+c_{n} z_{n}\right)+z_{n} d \leq-\sqrt{b_{m n}}\left|z_{n}\right| \bar{\varphi}^{\top} \tilde{\theta}+\sqrt{b_{m n}}\left|z_{n}\right| \bar{\varphi}^{\top} \hat{\theta}
$$

where

$$
\begin{aligned}
\tilde{\theta} & =\hat{\theta}-\theta \\
& =\hat{\theta}-\frac{1}{\sqrt{b_{m n}}}\left[\bar{\mu}_{1}, \cdots, \bar{\mu}_{j}, \mu_{d}, 1\right]^{\top}
\end{aligned}
$$

being $\hat{\theta}$ an adjusted parameter vector and $\tilde{\theta}$ an adjustment error. Using the property (3) in the term $\sqrt{b_{m n}}\left|z_{n}\right| \bar{\varphi}^{\top} \hat{\theta}$ of (25), we obtain:

$$
\begin{aligned}
& \sqrt{b_{m n}}\left|z_{n}\right|\left|\bar{\varphi}^{\top} \hat{\theta}\right| \leq \sqrt{\frac{3 C_{b v z}}{2}} \sqrt{\frac{2}{3 C_{b v z}}} \sqrt{|b|}\left|z_{n}\right|\left|\bar{\varphi}^{\top} \hat{\theta}\right| \\
& \text { where } C_{b v z}=(1 / 2) C_{b e}^{2}
\end{aligned}
$$


using the Young's inequality (cf. (Royden, 1988) pp. 123), we obtain:

$$
\sqrt{b_{m n}}\left|z_{n}\right|\left|\bar{\varphi}^{\top} \hat{\theta}\right| \leq \frac{3}{4} C_{b v z}+\frac{1}{3 C_{b v z}}|b| z_{n}^{2}\left(\bar{\varphi}^{\top} \hat{\theta}\right)^{2}
$$

Substituting (29) into (25), we obtain:

$$
\begin{aligned}
& z_{n} a^{\top} \gamma_{n}+z_{n}\left(\varphi_{n}+c_{n} z_{n}\right)+z_{n} d \\
& \quad \leq-\sqrt{b_{m n}}\left|z_{n}\right| \bar{\varphi}^{\top} \tilde{\theta}+\frac{3}{4} C_{b v z}+\frac{1}{3 C_{b v z}}|b| z_{n}^{2}\left(\bar{\varphi}^{\top} \hat{\theta}\right)^{2}
\end{aligned}
$$

Remark. We have proposed a new method to handle the unknown varying behavior of the control gain $b$, alternative to the current Nussbaum gain method. We parameterized the model term $z_{n} a^{\top} \gamma_{n}+$ $z_{n}\left(\varphi_{n}+c_{n} z_{n}\right)+z_{n} d$ in terms of adjustment error $\tilde{\theta}$ and adjusted parameter vector $\hat{\theta}$, and developed the following steps:

i) Introduce the constant $\sqrt{b_{m n}}$ in the parameterization, see (22).

ii) Introduce the inequality $\sqrt{b_{m n}} \leq \sqrt{|b|}$, see (27).

iii) Apply the Young's inequality to obtain b, see (29).

Recall that the value of $b_{m n}$ is not required to be known.

Substituting (30) into (20), we obtain:

$$
\begin{aligned}
& z_{n} \dot{z}_{n} \leq-c_{n} z_{n}^{2}+(3 / 4) C_{b v z}+b z_{n}\left(u+\frac{1}{3 C_{b v z}} \operatorname{sgn}(b) z_{n}\left(\bar{\varphi}^{\top} \hat{\theta}\right)^{2}\right) \\
& -\sqrt{b_{m n}}\left|z_{n}\right| \bar{\varphi}^{\top} \tilde{\theta}
\end{aligned}
$$

we choose the following control law:

$$
u=-\frac{1}{3 C_{b v z}} \operatorname{sgn}(b) z_{n}\left(\bar{\varphi}^{\top} \hat{\theta}\right)^{2}
$$

where $\bar{\varphi}, z_{n}$ are defined in (23), (16), respectively. Substituting (32) into (31), we obtain:

$$
z_{n} \dot{z}_{n} \leq-c_{n} z_{n}^{2}+(3 / 4) C_{b v z}-\sqrt{b_{m n}}\left|z_{n}\right| \bar{\varphi}^{\top} \tilde{\theta}
$$

To handle the effect of the constant $(3 / 4) C_{b v z}$, we formulate the following Lyapunov-like function:

$$
\begin{aligned}
& \bar{V}_{z}=\left\{\begin{array}{cr}
(1 / 2)\left(\sqrt{V_{z}}-\sqrt{C_{b v z}}\right)^{2} & \text { if } V_{z} \geq C_{b v z} \\
0 & \text { otherwise }
\end{array}\right. \\
& V_{z}=(1 / 2)\left(z_{1}^{2}+\cdots+z_{n}^{2}\right)
\end{aligned}
$$

where $C_{b v z}$ is defined in (28). We need the following properties: 
Proposition 5.1. The function $\bar{V}_{z}$ defined in (34) has the following properties:

$$
\begin{aligned}
& \text { i) } \bar{V}_{z} \geq 0 \\
& \text { ii) } V_{z} \leq 3 C_{b v z}+3 \bar{V}_{z} \\
& \text { iii) } \bar{V}_{z},\left(\partial \bar{V}_{z} / \partial V_{z}\right) \text { are continuous with respect to } V_{z}
\end{aligned}
$$

Proof. From (34) it follows that $\bar{V}_{z} \geq 0 \forall t \geq t_{0}$, the property i of proposition 5.1. In addition, from (34) it follows that

$$
V_{z} \leq\left(\sqrt{2 \bar{V}_{z}}+\sqrt{C_{b v z}}\right)^{2}
$$

Applying the Young's inequality (cf. (Royden, 1988) pp. 123), we obtain:

$$
V_{z}=C_{b v z}+2 \sqrt{C_{b v z}} \sqrt{2 \bar{V}_{z}}+2 \bar{V}_{z} \leq 3 C_{b v z}+3 \bar{V}_{z}
$$

This completes the proof of property ii. From (42) it follows that $\partial \bar{V}_{z} / \partial V_{z}=0$ if $V_{z}=C_{b v z}$ and that $\partial \bar{V}_{z} / \partial V_{z}$ is continuous. From (34) it follows that $\bar{V}_{z}$ is continuous. This completes the proof of property iii of proposition 5.1 .

Differentiating (34) with respect to time, we obtain:

$$
\begin{aligned}
& \frac{d \bar{V}_{z}}{d t}=\frac{\partial \bar{V}_{z}}{\partial V_{z}} \dot{V}_{z} \\
& \frac{\partial \bar{V}_{z}}{\partial V_{z}}=\left\{\begin{array}{cr}
(1 / 2)\left(1 / \sqrt{V_{z}}\right)\left(\sqrt{V_{z}}-\sqrt{C_{b v z}}\right) & \text { if } V_{z} \geq C_{b v z} \\
0 & \text { otherwise }
\end{array}\right.
\end{aligned}
$$

To compute $\dot{V}_{z}$, we differentiate (35) with respect to time: $\dot{V}_{z}=z_{1} \dot{z}_{1}+\cdots+z_{n} \dot{z}_{n}$. Introducing (11) and (15), we obtain

$$
\dot{V}_{z}=-c_{1} z_{1}^{2}+z_{1} z_{2}-c_{2} z_{2}^{2}+z_{2} z_{3}+\cdots+z_{n} \dot{z}_{n}
$$

substituting (33), we obtain:

$$
\begin{aligned}
\dot{V}_{z} \leq- & c_{1} z_{1}^{2}+z_{1} z_{2}-c_{2} z_{2}^{2}+z_{2} z_{3}+\cdots-c_{n} z_{n}^{2} \\
& +(3 / 4) C_{b v z}-\sqrt{b_{m n}}\left|z_{n}\right| \bar{\varphi}^{\top} \tilde{\theta}
\end{aligned}
$$

Provided that $c_{1} \geq 2, c_{2} \geq 2, \cdots, c_{n} \geq 2$ and completing the squares yields:

$$
\begin{aligned}
& -c_{1} z_{1}^{2}+z_{1} z_{2}-c_{2} z_{2}^{2}+z_{2} z_{3} \cdots-c_{n} z_{n}^{2} \\
& \quad \leq-z_{1}^{2}-(3 / 4) z_{2}^{2}+\cdots-(3 / 4) z_{n}^{2} \leq-(3 / 2) V_{z}
\end{aligned}
$$

substituting into (44), we obtain:

$$
\dot{V}_{z} \leq-(3 / 2) V_{z}+(3 / 4) C_{b v z}-\sqrt{b_{m n}}\left|z_{n}\right| \bar{\varphi}^{\top} \tilde{\theta}
$$


Since $\partial \bar{V}_{z} / \partial V_{z}$ is non-negative, we can multiply it into (45) without changing the direction of the inequality:

$$
\frac{\partial \bar{V}_{z}}{\partial V_{z}} \dot{V}_{z} \leq-(3 / 2) V_{z} \frac{\partial \bar{V}_{z}}{\partial V_{z}}+(3 / 4) C_{b v z} \frac{\partial \bar{V}_{z}}{\partial V_{z}}-\sqrt{b_{m n}}\left|z_{n}\right| \bar{\varphi}^{\top} \tilde{\theta} \frac{\partial \bar{V}_{z}}{\partial V_{z}}
$$

Substituting into (41), we obtain:

$$
\frac{d \bar{V}_{z}}{d t} \leq-(3 / 2) V_{z} \frac{\partial \bar{V}_{z}}{\partial V_{z}}+(3 / 4) C_{b v z} \frac{\partial \bar{V}_{z}}{\partial V_{z}}-\sqrt{b_{m n}}\left|z_{n}\right| \bar{\varphi}^{\top} \tilde{\theta} \frac{\partial \bar{V}_{z}}{\partial V_{z}}
$$

we choose the update law so as to reject the effect of the term involving the adjustment error $\tilde{\theta}$ :

$$
\dot{\hat{\theta}}=\Gamma \bar{\varphi}\left|z_{n}\right| \frac{\partial \bar{V}_{z}}{\partial V_{z}}
$$

where $\Gamma$ is a diagonal matrix whose diagonal elements are positive constants defined by the user, whereas $\bar{\varphi}, z_{n}, \partial \bar{V}_{z} / \partial V_{z}$ are defined in (23), (16), (42), respectively.

Remark. So far, we have developed the controller, which involves the control law (32) and the update law (48). Other parameters necessary for its implementation are: $V_{z}$ defined in (35); $z_{1}, z_{2}, \cdots, z_{n}$ defined in (9), (12), $\cdots,(16)$, respectively; $C_{b v z}$ defined in (28). Recall that $c_{1} \geq 2, \cdots, c_{n} \geq 2$ are user-defined positive constants.

Remark. The control and update laws stated in (32) and (48) have the following features:

i) The control law uses the adjusted parameter vector $\hat{\theta}$, so that it does not rely on upper or lower bounds of the plant coefficients, i.e. $\bar{\mu}_{1}, \cdots, \bar{\mu}_{j}, \mu_{d}, b_{m n}$, and excessive control effort is also avoided.

ii) Additional states are not required to handle the unknown varying behavior of the control gain, what is an important benefit with respect to closely related schemes that use the Nussbaum gain method.

iii) The control and update laws do not involve discontinuous signals. In fact, the vectorial field of the closed loop system is Lipschitz continuous, so that trajectory unicity is preserved.

\section{Boundedness analysis}

In this section we prove that the closed loop signals $z_{1}, \cdots, z_{n}, \hat{\theta}, u$ are bounded if the developed controller is applied.

Theorem 6.1. Boundedness of the closed loop signals. Consider the plant (1) subject to assumptions $A i$ to Av; the signals $z_{1}, \cdots, z_{n}$ defined in (9), (12) and (16); the signals $\bar{\varphi}, V_{z}, \partial \bar{V}_{z} / \partial V_{z}$, $C_{b v z}$ defined in (23), (35), (42) and (28), respectively. If the controller (32), (48) is applied, then the signals $z_{1}, \cdots, z_{n}, \hat{\theta}$, and $u$ remain bounded and $|e|$ is upper bounded as follows:

$$
|e| \leq \sqrt{2}\left(\sqrt{C_{b v z}}+\sqrt{2 V\left(\bar{x}\left(t_{o}\right)\right)}\right)^{2}
$$

Proof. We choose the following Lyapunov-like function:

$$
V(\bar{x}(t))=\bar{V}_{z}+V_{\theta}
$$




$$
\begin{aligned}
& V_{\theta}=(1 / 2) \sqrt{b_{m n}} \tilde{\theta}^{\top} \Gamma^{-1} \tilde{\theta} \\
& \bar{x}(t)=\left[\begin{array}{ll}
z_{1}, & \ldots, \\
z_{n}, & \tilde{\theta}^{\top}
\end{array}\right]^{\top}
\end{aligned}
$$

where $\bar{V}_{z}$ is defined in (34) and $\tilde{\theta}$ in (26). The time derivative of $V_{\theta}$ is:

$$
\dot{V}_{\theta}=(1 / 2) \sqrt{b_{m n}}\left(\dot{\tilde{\theta}}^{\top} \Gamma^{-1} \tilde{\theta}+\tilde{\theta}^{\top} \Gamma^{-1} \dot{\tilde{\theta}}\right)
$$

Since $\Gamma$ is diagonal, then $\Gamma^{-1}$ is diagonal, $\left(\Gamma^{-1}\right)^{\top}=\Gamma^{-1}, \dot{\tilde{\theta}}^{\top} \Gamma^{-1} \tilde{\theta}=\tilde{\theta}^{\top} \Gamma^{-1} \dot{\tilde{\theta}}$. In view of this and the update law (48), we have:

$$
\dot{V}_{\theta}=\sqrt{b_{m n}} \tilde{\theta}^{\top} \Gamma^{-1} \dot{\hat{\theta}}=\sqrt{b_{m n}} \tilde{\theta}^{\top} \bar{\varphi}\left|z_{n}\right| \frac{\partial \bar{V}_{z}}{\partial V_{z}}
$$

Differentiating (50) with respect to time, we obtain: $\dot{V}=\dot{\bar{V}}_{z}+\dot{V}_{\theta}$. Substituting equations (47) and (54), we obtain:

$$
\begin{aligned}
\dot{V} & \leq-(3 / 2) V_{z} \frac{\partial \bar{V}_{z}}{\partial V_{z}}+(3 / 4) C_{b v z} \frac{\partial \bar{V}_{z}}{\partial V_{z}} \\
& =-\frac{3}{2} \frac{\partial \bar{V}_{z}}{\partial V_{z}}\left(\frac{V_{z}}{2}+\frac{V_{z}}{2}-\frac{C_{b v z}}{2}\right)
\end{aligned}
$$

From (42) if follows that

$$
\begin{aligned}
& \partial \bar{V}_{z} / \partial V_{z}=0 \text { for } V_{z} \leq C_{b v z} \\
& \partial \bar{V}_{z} / \partial V_{z}>0 \text { for } V_{z}>C_{b v z} .
\end{aligned}
$$

In view of this and (55), we obtain:

$$
\begin{aligned}
& \dot{V} \leq-\frac{3}{2} \frac{\partial \bar{V}_{z}}{\partial V_{z}}\left(\frac{V_{z}}{2}\right) \text { if } V_{z} \geq C_{b v z} \\
& \dot{V} \leq 0=-\frac{3}{2} \frac{\partial \bar{V}_{z}}{\partial V_{z}}\left(\frac{V_{z}}{2}\right) \text { if } V_{z}<C_{b v z} \\
& \Rightarrow \dot{V} \leq-\frac{3}{4} \frac{\partial \bar{V}_{z}}{\partial V_{z}} V_{z} \leq 0
\end{aligned}
$$

Thus, $\dot{V}+0 V \leq 0$. Using the Lemma in (Slotine \& Li, 1991) pp. 91, we obtain:

$$
V(\bar{x}(t)) \leq V\left(\bar{x}\left(t_{o}\right)\right) \exp (-0 t)=V\left(\bar{x}\left(t_{o}\right)\right)
$$

where

$$
\begin{aligned}
& V\left(\bar{x}\left(t_{o}\right)\right)=\bar{V}_{z o}+V_{\theta o} \\
& \bar{V}_{z o}=\left\{\begin{array}{cr}
(1 / 2)\left(\sqrt{V_{z o}}-\sqrt{C_{b v z}}\right)^{2} \text { if } V_{z o} \geq C_{b v z} \\
0 & \text { otherwise }
\end{array}\right. \\
& V_{z o}=(1 / 2)\left(z_{1}\left(t_{o}\right)^{2}+\cdots+z_{n}\left(t_{o}\right)^{2}\right) \\
& V_{\theta o}=(1 / 2) \sqrt{b_{m n}}\left(\hat{\theta}\left(t_{o}\right)-\theta\right)^{\top} \Gamma^{-1}\left(\hat{\theta}\left(t_{o}\right)-\theta\right)
\end{aligned}
$$


Since $V(\bar{x}(t)) \geq 0$, we have: $0 \leq V(\bar{x}(t)) \leq V\left(\bar{x}\left(t_{0}\right)\right)$. Introducing the definition (50), we obtain

$$
\begin{aligned}
& \bar{V}_{z}+V_{\theta} \leq V\left(\bar{x}\left(t_{o}\right)\right) \\
& \Rightarrow \bar{V}_{z} \leq V\left(\bar{x}\left(t_{o}\right)\right), \quad V_{\theta} \leq V\left(\bar{x}\left(t_{o}\right)\right)
\end{aligned}
$$

Thus, it follows from (51) that $\tilde{\theta} \in L_{\infty}$, and consequently $\hat{\theta} \in L_{\infty}$. The inequality $\bar{V}_{z} \leq V\left(\bar{x}\left(t_{0}\right)\right)$ implies that the tracking error $e$ is bounded, as we show hereafter. We begin by solving (34) for $V_{z}$ :

$$
\begin{aligned}
& V_{z}=\left(\sqrt{C_{b v z}}+\sqrt{2 \bar{V}_{z}}\right)^{2} \text { if } \bar{V}_{z}>0 \\
& V_{z} \leq C_{b v z} \text { if } \bar{V}_{z}=0
\end{aligned}
$$

Using the inequality $\bar{V}_{z} \leq V\left(\bar{x}\left(t_{o}\right)\right)$, we obtain:

$$
\begin{aligned}
& V_{z} \leq\left(\sqrt{C_{b v z}}+\sqrt{2 V\left(\bar{x}\left(t_{o}\right)\right)}\right)^{2} \text { if } \bar{V}_{z}>0 \\
& V_{z} \leq C_{b v z} \leq\left(\sqrt{C_{b v z}}+\sqrt{2 V\left(\bar{x}\left(t_{o}\right)\right)}\right)^{2} \text { if } \bar{V}_{z}=0
\end{aligned}
$$

combining both inequalities, we obtain:

$$
V_{z} \leq\left(\sqrt{C_{b v z}}+\sqrt{2 V\left(\bar{x}\left(t_{o}\right)\right)}\right)^{2}
$$

Introducing the definition (35), we obtain:

$$
\sqrt{z_{1}^{2}+\cdots+z_{n}^{2}} \leq \sqrt{2}\left(\sqrt{C_{b v z}}+\sqrt{2 V\left(\bar{x}\left(t_{o}\right)\right)}\right)^{2}
$$

so that $z_{1} \in L_{\infty}, \cdots, z_{n} \in L_{\infty}$. Since $e^{2}=z_{1}^{2} \leq z_{1}^{2}+\cdots+z_{n}^{2}$, we obtain:

$$
|e| \leq \sqrt{2}\left(\sqrt{C_{b v z}}+\sqrt{2 V\left(\bar{x}\left(t_{o}\right)\right)}\right)^{2}
$$

which indicates the upper bound for the tracking error $e$.

Remark. Notice that this upper bound does not involve integral terms, what is an important advantage with respect to the Nussbaum Gain method, see (Su et al., 2009), (Feng, Hong, Chen E Su, 2008), (Feng et al., 2006), (Ge E Wang, 2003), (Feng et al., 2007), (Feng, Su E Hong, 2008), (Ren et al., 2008).

Now, we proceed to show the boundedness of $u$. From (9), (12), (16) it follows that $x_{1} \in L_{\infty}$, $x_{2} \in L_{\infty}, \cdots, x_{n} \in L_{\infty}$. Therefore, $\gamma_{n} \in L_{\infty}$. It follows from (23) that $\bar{\varphi} \in L_{\infty}$. From (32) it follows that $u \in L_{\infty}$. This completes the proof. 


\section{Convergence analysis}

In this section we prove that if the developed controller is applied, then the signal $z_{1}$ converges asymptotically to $\Omega_{z}$, where $\Omega_{z}=\left\{z_{1}:\left|z_{1}\right| \leq C_{b e}\right\}$.

Theorem 7.1. Convergence of the tracking error. Consider the plant (1) subject to assumptions Ai to Av; the signals $z_{1}, \cdots, z_{n}$ defined in (9), (12) and (16); the signals $\bar{\varphi}, V_{z}, \partial \bar{V}_{z} / \partial V_{z}, C_{b v z}$ defined in (23), (35), (42) and (28), respectively. If the controller (32), (48) is applied, then the signal $z_{1}$ converges asymptotically to $\Omega_{z}$, where $\Omega_{z}=\left\{z_{1}:\left|z_{1}\right| \leq C_{b e}\right\}$.

Proof. In view of (42), equation (58) can be rewritten as:

$$
\begin{aligned}
& \dot{V} \leq-f_{d} \leq 0 \\
& f_{d}=\left\{\begin{array}{cr}
(3 / 8)\left(\sqrt{V_{z}}\right)\left(\sqrt{V_{z}}-\sqrt{C_{b v z}}\right) & \text { if } V_{z} \geq C_{b v z} \\
0 & \text { otherwise }
\end{array}\right.
\end{aligned}
$$

The derivative $\partial f_{d} / \partial V_{z}$ is not continuous, as it involves an abrupt change at $V=C_{b v z}$. Thus, the Barbalat's Lemma can not be applied on $f_{d}$. To remedy that, we shall express (73) in terms of a function with continuous derivative:

$$
\begin{aligned}
& \dot{V} \leq-f_{d} \leq-f_{g} \leq 0 \\
& f_{g}=\left\{\begin{array}{cr}
(3 / 8)\left(\sqrt{V_{z}}-\sqrt{C_{b v z}}\right)^{2} & \text { if } V_{z} \geq C_{b v z} \\
0 & \text { otherwise }
\end{array}\right.
\end{aligned}
$$

Arranging and integrating (75), we obtain:

$$
\begin{aligned}
& f_{g} \leq-\dot{V} \\
& \int_{t o}^{t} f_{g} d \tau \leq V\left(\bar{x}\left(t_{o}\right)\right)-V(\bar{x}(t)) \\
& V(\bar{x}(t))+\int_{t o}^{t} f_{g} d \tau \leq V\left(\bar{x}\left(t_{o}\right)\right)
\end{aligned}
$$

Thus, $f_{g} \in L_{1}$. We have to prove that $f_{g} \in L_{\infty}, \dot{f}_{g} \in L_{\infty}$ to apply the Barbalat's Lemma. Since $V_{z} \in L_{\infty}$, it follows from (76) that $f_{g} \in L_{\infty}$. Differentiating (76) with respect to time, we obtain:

$$
\begin{aligned}
& \dot{f}_{g}=\frac{\partial f_{g}}{\partial V_{z}} \dot{V}_{z} \\
& \frac{\partial f_{g}}{\partial V_{z}}=\left\{\begin{array}{cc}
(3 / 8)\left(1 / \sqrt{V_{z}}\right)\left(\sqrt{V_{z}}-\sqrt{C_{b v z}}\right) & \text { if } V_{z} \geq C_{b v z} \\
0 & \text { otherwise }
\end{array}\right.
\end{aligned}
$$

Notice that $\partial f_{g} / \partial V_{z}$ is continuous. Since $V_{z} \in L_{\infty}$, then $\partial f_{g} / \partial V_{z} \in L_{\infty}$. Since $z_{1} \in L_{\infty}, \cdots$, $z_{n} \in L_{\infty}$, it follows from (11), (15) that $\dot{z}_{1} \in L_{\infty}, \cdots, \dot{z}_{n-1} \in L_{\infty}$. Since $u \in L_{\infty}$, it follows from (17) that $\dot{z}_{n} \in L_{\infty}$. Therefore, from (43) it follows that $\dot{V}_{z} \in L_{\infty}$.

So far we have proved that $\partial f_{g} / \partial V_{z} \in L_{\infty}$ and $\dot{V}_{z} \in L_{\infty}$, so that it follows from (78) that $\dot{f}_{g} \in L_{\infty}$. In view of $f_{g} \in L_{\infty}, \dot{f}_{g} \in L_{\infty}$, application of Barbalat's Lemma (cf. (Ioannou \& Sun, 1996) pp. 76), then indicates that $f_{g}$ converges asymptotically to zero. Hence, from (76) it follows that $V_{z}$ converges to $\Omega_{v z}$, where $\Omega_{v z}=\left\{V_{z}: V_{z} \leq C_{b v z}\right\}$. From the definition (35), 
it follows that $z_{1}$ converges asymptotically to $\Omega_{z}$, where $\Omega_{z}=\left\{z_{1}:\left|z_{1}\right| \leq \sqrt{2 C_{b v z}}\right\}$. Since $C_{b v z}=(1 / 2) C_{b e}^{2}$, it follows that $\Omega_{z}=\left\{z_{1}:\left|z_{1}\right| \leq C_{b e}\right\}$. This completes the proof.

\section{Simulation example}

Consider the following case of the plant (1):

$$
\begin{aligned}
& \ddot{y}=\gamma_{2}^{\top} a+b u+d \\
& \gamma_{2}=\left[\begin{array}{ll}
\dot{y}, & y
\end{array}\right]^{\top}, a=\left[\begin{array}{ll}
a_{1}, & a_{2}
\end{array}\right]^{\top} \\
& a_{1}=-2(1+0.1 \sin (2(\pi / 8) t)), a_{2}=-1(1+0.1 \sin (2(\pi / 5) t)) \\
& b=2(1+0.1 \sin ((2 \pi / 11) t))+0.6|y| \\
& d=-0.2(1+0.1 \sin ((2 \pi / 7) t)) y
\end{aligned}
$$

The aim is that $y$ converges towards $y_{d}$, with a threshold of 0.1 . In figure 1 we present a simulation block diagram for the example.

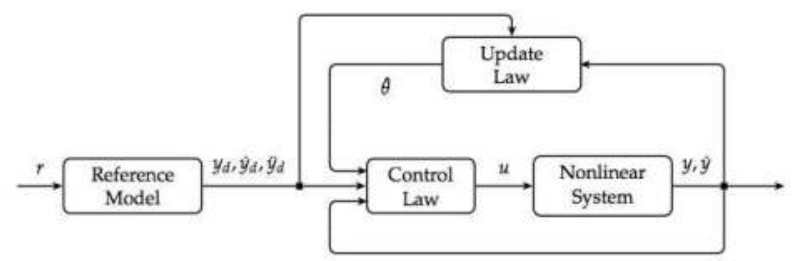

Fig. 1. Simulation block diagram.

The properties Ai, Aiv, Av of section 2 are analyzed at the following. From (82) it follows that $a_{1}, d, b$ are bounded as:

$$
\begin{aligned}
& \left|a_{1}\right| \leq 2(1.1)=2.2, \quad\left|a_{2}\right| \leq 1(1.1)=1.1 \\
& |d| \leq 0.2(1.1)|y|=0.22|y| \\
& |b| \geq 2(0.9)=1.8>0
\end{aligned}
$$

Hence, the upper bounds of $a_{1}, a_{2}, d$, and the lower bound of $b$, are:

$$
\begin{aligned}
& \left|a_{1}\right| \leq \bar{\mu}_{1}=2.2,\left|a_{2}\right| \leq \bar{\mu}_{2}=1.1,|b| \geq b_{m n}=1.8 \\
& |d| \leq \mu_{d} f_{d}, \mu_{d}=0.22, f_{d}=|y|
\end{aligned}
$$

where $f_{d}$ is not constant and known, whereas $b_{m n}, \bar{\mu}_{1}, \bar{\mu}_{2}, \mu_{d}, b_{m n}$ are positive, constant and unknown to the controller. From (86), (87) it follows that assumptions Ai, Aiv, Av of section 2 are satisfied.

The procedure of section 4 is followed in order to establish the terms involved in the control and update laws, mentioned in remark 5. Eq. (80) can be rewritten as:

$$
\begin{aligned}
& \dot{x}_{1}=x_{2} \\
& \dot{x}_{2}=\gamma_{2}^{\top} a+b u+d \\
& x_{1}=y, \quad x_{2}=\dot{y}, \quad n=2
\end{aligned}
$$


since $n=2$, the state transformation based on the backstepping procedure involves the steps $0,1,2$.

Step 0. Let

$$
z_{1}=e=y-y_{d}=x_{1}-y_{d}
$$

as in (9).

Step 1. Differentiating (91) with respect to time and arranging, yields:

$$
\begin{aligned}
& \dot{z}_{1}=-c_{1} z_{1}+z_{2} \\
& z_{2}=x_{2}+c_{1} z_{1}-\dot{y}_{d}
\end{aligned}
$$

as in (11), (12).

Step 2. Since $n=2$, the second step is the last one. Differentiating (93) with respect to time, using (89) and arranging, yields:

$$
\begin{aligned}
\dot{z}_{2} & =\dot{x}_{2}+c_{1} \dot{z}_{1}-\ddot{y}_{d} \\
& =\gamma_{2}^{\top} a+b u+d+c_{1} \dot{z}_{1}-\ddot{y}_{d} \\
& =\gamma_{2}^{\top} a+b u+d+c_{1}\left(x_{2}+\varphi_{1}\right)-\ddot{y}_{d}
\end{aligned}
$$

using the definitions (91), (93), yields:

$$
\begin{aligned}
& \dot{z}_{2}=\gamma_{2}^{\top} a+b u+d+\varphi_{2} \\
& \varphi_{2}=c_{1}\left(z_{2}-c_{1} z_{1}\right)-\ddot{y}_{d}
\end{aligned}
$$

notice that the form of (95), (96) is that of (17), (18), respectively. This completes the state transformation based on the backstepping procedure.

The parameters defined above can be summarized as:

$$
\begin{aligned}
z_{1} & =y-y_{d} \\
z_{2} & =x_{2}+c_{1} z_{1}-\dot{y}_{d} \\
x_{1} & =y, x_{2}=\dot{y}_{d} \\
\varphi_{1} & =-\dot{y}_{d} \\
\varphi_{2} & =c_{1}\left(z_{2}-c_{1} z_{1}\right)-\ddot{y}_{d}
\end{aligned}
$$

According to remark 5, it remains to define $\bar{\varphi}, V_{z}$. From (81), definition (23) and $n=2$, it follows that

$$
\bar{\varphi}=\left[\left|\gamma_{2[1]}\right|,\left|\gamma_{2[2]}\right|, f_{d},\left|\varphi_{2}+c_{2} z_{2}\right|\right]^{\top}=\left[|\dot{y}|,|y|, f_{d},\left|\varphi_{2}+c_{2} z_{2}\right|\right]^{\top}
$$

From (35) and $n=2$ it follows that

$$
V_{z}=(1 / 2)\left(z_{1}^{2}+z_{2}^{2}\right)
$$


Expressions (97) to (103) allow to define the control and update law. From (32), (48), (82) and $n=2$ it follows that

$$
\begin{aligned}
& \operatorname{sgn}(b)=+1 \\
& u=-\frac{1}{3 C_{b v z}} z_{2}\left(\bar{\varphi}^{\top} \hat{\theta}\right)^{2} \\
& \dot{\hat{\theta}}=\Gamma \bar{\varphi}\left|z_{2}\right| \frac{\partial \bar{V}_{z}}{\partial V_{z}}
\end{aligned}
$$

the main parameters needed to compute $u$ and $\hat{\theta}$ are: $\bar{\varphi}$ (102), $\varphi_{2}$ (101), $C_{b v z}(28), z_{2}(98), z_{1}$ (97), $\partial \bar{V}_{z} / \partial V_{z}(42), V_{z}$ (103). In addition, $\Gamma$ is a diagonal matrix whose diagonal elements are positive constants defined by the user.
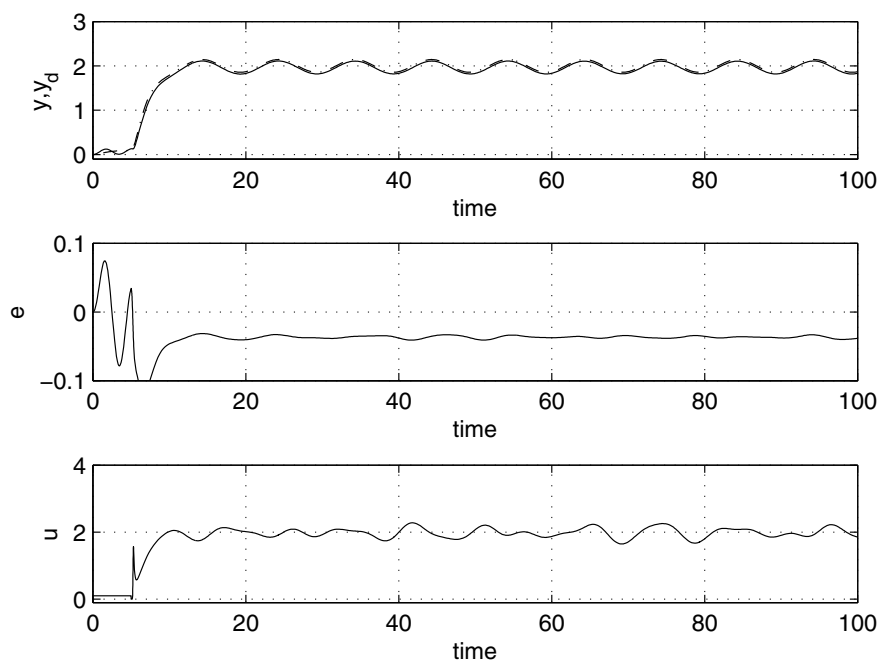

Fig. 2. Example 1, upper: output $y$ (continuous line), desired output $y_{d}$ (dash-dot line); middle: tracking error $e$; lower: control input $u$.

Since the aim is that $y$ converges towards $y_{d}$, with a threshold of 0.1 , we set $C_{b e}=0.1$. We use the reference model (5) with $y_{d}\left(t_{0}\right)=y\left(t_{0}\right), \dot{y}_{d}\left(t_{0}\right)=0, a_{m, 1}=1, a_{m, 0}=1$. We use the following parameter values for the control and update laws: $c_{1}=2, c_{2}=2$, $\Gamma=\operatorname{diag}\{1,1,1,1\}$.

The results are shown in figures 2 and 3 . We have choosen $y_{d}\left(t_{0}\right) \approx y\left(t_{0}\right)$ in order to obtain a rapid convergence of $y$ towards $y_{d}$. Figure 2 shows that. i) the tracking error $e$ converges asymptotically towards $\Omega_{e}=\{e:|e| \leq 0.1\}$. ii) The output $y$ converges towards $y_{d}$ with threshold 0.1 without large transient differences. Figure 3 shows that $\hat{\theta}_{1}, \ldots, \hat{\theta}_{4}$ are not decreasing with respect to time. This occurs because $\dot{\hat{\theta}}$ is non-negative. The procedure for the sample plant (80) is simpler in comparison with adapive controllers that use the Nussbaum gain method. 

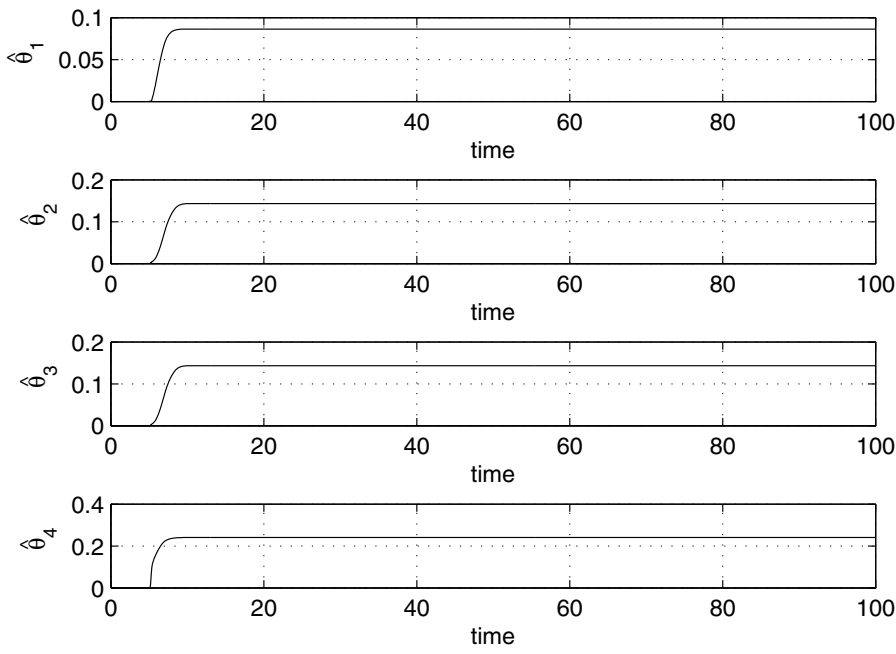

Fig. 3. Example 1, entries of the updated parameter vector $\hat{\theta}$, from upper to lower: $\hat{\theta}_{1} ; \hat{\theta}_{2}, \hat{\theta}_{3}$, $\hat{\theta}_{4}$.

\section{Acknowledgements}

A. Rincon acknowledges financial support provided by "Programa de becas para estudiantes sobresalientes de posgrado", Universidad Nacional de Colombia - vicerrectoría de Investigación. This work was partially supported by Universidad Nacional de Colombia Manizales, project 12475, Vicerrectoría de Investigación, DIMA.

\section{References}

Bashash, S. \& Jalili, N. (2009). Robust adaptive control of coupled parallel piezo-flexural nanopositioning stages, IEEE/ASME Transactions on Mechatronics Vol. 14(No. 1): 11-20.

Bechlioulis, C. \& Rovithakis, G. (2009). Adaptive control with guaranteed transient and steady state tracking error bounds for strict feedback systems, Automatica Vol. 45(No. 2): 532-538.

Cao, C. \& Hovakimyan, N. (2006). Design and analysis of a novel $l_{1}$ adaptive controller, part i: control signal and asymptotic stability, Proceedings of the 2006 American Control Conference, Publisher, Minneapolis (USA), pp. 3397-3402.

Cao, C. \& Hovakimyan, N. (2008a). Design and analysis of a novel adaptive control architecture with guaranteed transient performance, IEEE Transactions on Automatic Control Vol. 53(No. 2): 586-591.

Cao, C. \& Hovakimyan, N. (2008b). $l_{1}$ adaptive output feedback controller for systems of unknown dimension, IEEE Transactions on Automatic Control Vol. 53(No. 3): 815-821.

Chen, B., Liu, X., Liu, K. \& Lin, C. (2009). Novel adaptive neural control design for nonlinear mimo time-delay systems, Automatica Vol. 45(No. 6): 1554-1560. 
Chen, C., Lin, C. \& Chen, T. (2008). Intelligent adaptive control for mimo uncertain nonlinear systems, Expert Systems with Applications Vol. 35(No. 3): 865 - 877.

Chen, W. (2009). Adaptive backstepping dynamic surface control for systems with periodic disturbances using neural networks, IET Control Theory and Applications Vol. 3(No. 10): 1383-1394.

Dobrokhodov, V., Kitsios, I., Kaminer, I. \& Jones, K. (2008). Flight validation of a metrics driven $l_{1}$ adaptive control, Proceedings of AIAA Guidance, Navigation and Control Conference and Exhibit, Publisher, Honolulu (USA), pp. 1-22.

Du, H., Ge, S. \& Liu, J. (2010). Adaptive neural network output feedback control for a class of non-afine non-linear systems with unmodelled dynamics, IET Control Theory and Applications Vol. 5(No. 3): 465-477.

Feng, Y., Hong, H., Chen, X. \& Su, C. (2008). Robust adaptive controller design for a class of nonlinear systems preceded by generalized prandtl-ishlinskii hysteresis representation, Proceedings of 7 th World Congress on Intelligent Control and Automation, Publisher, Chongqing (China), pp. 382-387.

Feng, Y., Hu, Y. \& Su, C. (2006). Robust adaptive control for a class of perturbed strict-feedback nonlinear systems with unknown prandtl-ishlinskii hysteresis, Proceedings of the 2006 IEEE International Symposium on Intelligent Control, Publisher, Munich (Germany), pp. 106-111.

Feng, Y., Su, C. \& Hong, H. (2008). Universal contruction of robust adaptive control laws for a class of nonlinear systems preceded by generalized prandtl-ishlinskii representation, Proceedings of the 3rd IEEE Conference on Industrial Electronics and Applications, Publisher, Singapore, pp. 153-158.

Feng, Y., Su, C., Hong, H. \& Ge, S. (2007). Robust adaptive control for a class of nonlinear systems with generalized prandtl-ishlinskii hysteresis, Proceedings of the 46th IEEE Conference on Decision and Control, Publisher, New Orleans (LA, USA), pp. 4833-4838.

Ge, S. \& Tee, K. (2007). Approximation-based control of nonlinear mimo time-delay systems, Automatica Vol. 43(No. 1): 31-43.

Ge, S. \& Wang, J. (2003). Robust adaptive tracking for time-varying uncertain nonlinear systems with unknown control coefficients, IEEE Transactions on Automatic Control Vol. 48(No. 8): 1463-1469.

Ho, H., Wong, Y. \& Rad, A. (2009). Adaptive fuzzy sliding mode control with chattering elimination for nonlinear siso systems, Simulation Modelling Practice and Theory Vol. 17(No. 7): 1199-1210.

Hong, Y. \& Yao, B. (2007). A globally stable saturated desired compensation adaptive robust control for linear motor systems with comparative experiments, Automatica Vol. 43(No. 10): 1840-1848.

Hsu, C., Lin, C. \& Lee, T. (2006). Wavelet adaptive backstepping control for a class of nonlinear systems, IEEE Transactions on Neural Networks Vol. 17(No. 5): 1175-1183.

Huang, A. \& Kuo, Y. (2001). Sliding control of non-linear systems containing time-varying uncertainties with unknown bounds, International Journal of Control Vol. 74(No. 3): $252-264$.

Ioannou, P. \& Sun, J. (1996). Robust Adaptive Control, Prentice-Hall PTR Upper Saddle River, New Jersey. 
Jiang, Z. \& Hill, D. (1999). A robust adaptive backstepping scheme for nonlinear systems with unmodeled dynamics, IEEE Transactions on Automatic Control Vol. 44(No. 9): 17051711.

Kanellakopoulos, I., Kokotović, P. \& Morse, A. (1991). Systematic design of adaptive controllers for feeedback linearizable systems, IEEE Transactions on Automatic Control Vol. 36(No. 11): 1241 - 1253.

Koo, K. (2001). Stable adaptive fuzzy controller with time-varying dead zone, Fuzzy Sets and Systems Vol. 121(No. 1): 161-168.

Labiod, S. \& Guerra, T. (2007). Adaptive fuzzy control of a class of siso nonaffine nonlinear systems, Fuzzy Sets and Systems Vol. 158(No. 10): 1126 - 1137.

Li, D. \& Hovakimyan, N. (2008). Filter design for feedback-loop trade-off of $l_{1}$ adaptive controller: a linear matrix inequality approach, Proceedings of IAA Guidance, Navigation and Control Conference and Exhibit, Publisher, Honolulu (USA), pp. 1-12.

Li, Y., Qiang, S., Zhuang, X. \& Kaynak, O. (2004). Robust and adaptive backstepping control for nonlinear systems using rbf neural networks, IEEE Transactions on Neural Networks Vol. 15(No. 3): 693-701.

Liu, C. \& Tong, S. (2010). Fuzzy adaptive decentralized control for nonlinear systems with unknown high-frequency gain sign based on k-filter, Proceedings of Control and Decision Conference, Publisher, China, pp. 1576-1581.

Nakanishi, J., Farrell, J. \& Schaal, S. (2005). Composite adaptive control with locally weighted statistical learning, Neural Networks Vol. 18(No. 1): 71-90.

Park, B., Yoo, S., Park, J. \& Choi, Y. (2009). Adaptive neural sliding mode control of nonholonomic wheeled mobile robots with model uncertainty, IEEE Transactions on Control Systems Technology Vol. 17(No. 1): 201-214.

Psillakis, H. (2010). Further results on the use of nussbaum gains in adaptive neural network control, IEEE Transactions on Automatic Control Vol. 55(No. 12): 2841-2846.

Ren, B., Ge, S., Lee, T. \& Su, C. (2008). Adaptive neural control for uncertain nonlinear systems in pure-feedback form with hysteresis input, Proceedings of the 47th IEEE Conference on Decision and Control, Publisher, Cancun (Mexico), pp. 86-91.

Royden, H. (1988). Real Analysis, Prentice Hall Upper Saddle River, New Jersey.

Slotine, J. \& Li, W. (1991). Applied Nonlinear Control, Prentice Hall Englewood Cliffs, New Jersey.

Su, C., Feng, Y., Hong, H. \& Chen, X. (2009). Adaptive control of system involving complex hysteretic nonlinearities: a generalised prandtl-ishlinskii modelling approach, International Journal of Control Vol. 82(No. 10): 1786-1793.

Tong, S., Liu, C. \& Li, Y. (2010). Fuzzy-adaptive decentralized output-feedback control for large-scale nonlinear systems with dynnamical uncertainties, IEEE Transaction on Fuzzy Systems Vol. 18(No. 5): 1-1.

Tong, S., Tang, J. \& Wang., T. (2000). Fuzzy adaptive control of multivariable nonlinear systems, Fuzzy Sets and Systems Vol. 111(No. 2): 153-167.

Wang, X., Su, C. \& Hong, H. (2004). Robust adaptive control of a class of nonlinear systems with unknown dead-zone, Automatica Vol. 40(No. 3): 407-413.

Wen, C., Zhou, J. \& Wang, W. (2009). Decentralized adaptive backst epping stabilization of interconnected systems with dynamic input and output interactions, Automatica Vol. 45(No. 1): 55-67. 
Yao, B. (1997). High performance adaptive robust control of nonlinear systems: a general framework and new schemes, Proceedings of the 36th Conference on Decision and Control, Publisher, San Diego (California, USA), pp. 2489-2494.

Yao, B. \& Tomizuka, M. (1994). Robust adaptive sliding mode control of manipulators with guaranteed transient performance, Proceedings of the American Control Conference, Publisher, Baltimore (Maryland, USA), pp. 1176-1180.

Yao, B. \& Tomizuka, M. (1997). Adaptive robust control of siso nonlinear systems in a semi-strict feedback form, Automatica Vol. 33(No. 5): 893-900.

Yousef, H. \& Wahba, M. (2009). Adaptive fuzzy mimo control of induction motors, Expert Systems with Applications Vol. 36(No. 3): 4171-4175.

Zhang, T. \& Ge, S. (2009). Adaptive neural network tracking control of mimo nonlinear systems with unknown dead zones and control directions, IEEE Transactions on Neural Networks Vol. 20(No. 3): 483-497.

Zhou, J., Wen, C. \& Wang, W. (2009). Adaptive backstepping control of uncertain systems with unknown input time-delay, Automatica Vol. 45(No. 6): 1415-1422.

Zhou, J., Wen, C. \& Zhang, Y. (2004). Adaptive backstepping control of a class of uncertain nonlinear systems with unknown backlash-like hysteresis, IEEE Transactions on Automatic Control Vol. 49(No. 10): 1751-1757. 


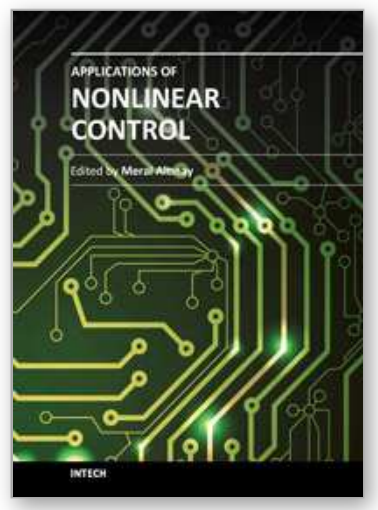

\author{
Applications of Nonlinear Control \\ Edited by Dr. Meral Altınay
}

ISBN 978-953-51-0656-2

Hard cover, 202 pages

Publisher InTech

Published online 13, June, 2012

Published in print edition June, 2012

A trend of inverstigation of Nonlinear Control Systems has been present over the last few decades. As a result the methods for its analysis and design have improved rapidly. This book includes nonlinear design topics such as Feedback Linearization, Lyapunov Based Control, Adaptive Control, Optimal Control and Robust Control. All chapters discuss different applications that are basically independent of each other. The book will provide the reader with information on modern control techniques and results which cover a very wide application area. Each chapter attempts to demonstrate how one would apply these techniques to real-world systems through both simulations and experimental settings.

\title{
How to reference
}

In order to correctly reference this scholarly work, feel free to copy and paste the following:

A. Rincon, F. Angulo and G. Osorio (2012). A Robust State Feedback Adaptive Controller with Improved Transient Tracking Error Bounds for Plants with Unknown Varying Control Gain, Applications of Nonlinear Control, Dr. Meral Altınay (Ed.), ISBN: 978-953-51-0656-2, InTech, Available from:

http://www.intechopen.com/books/applications-of-nonlinear-control/a-robust-state-feedback-adaptivecontroller-with-improved-transient-tracking-error-bounds-for-pl

\section{INTECH}

open science | open minds

\section{InTech Europe}

University Campus STeP Ri

Slavka Krautzeka 83/A

51000 Rijeka, Croatia

Phone: +385 (51) 770447

Fax: +385 (51) 686166

www.intechopen.com

\section{InTech China}

Unit 405, Office Block, Hotel Equatorial Shanghai

No.65, Yan An Road (West), Shanghai, 200040, China 中国上海市延安西路65号上海国际贵都大饭店办公楼 405 单元

Phone: +86-21-62489820

Fax: +86-21-62489821 
(C) 2012 The Author(s). Licensee IntechOpen. This is an open access article distributed under the terms of the Creative Commons Attribution 3.0 License, which permits unrestricted use, distribution, and reproduction in any medium, provided the original work is properly cited. 